\title{
ASPECTOS BIOLÓGICOS DE DIAPTERUS RHOMBEUS (CUVIER) (TELEOSTEI, GERREIDAE) NA BAÍA DE GUARATUBA, PARANÁ, BRASIL
}

\author{
Paulo de Tarso da Cunha Chaves ${ }^{2,3}$ \\ Gislaine Otto ${ }^{2,4}$
}

\begin{abstract}
Biological. aspects of Diapterus RHombeus (Cuvier) (Teleostei, Gerreidae) at Guaratuba Bay, Paraná, Brazil. Diapterus rhombeus (Cuvier, 1829 ) is one of the most common Gerreidae species in the estuarine region at the Guaratuba Bay, Southern Brazil. Based on studies developed between July, 1993 and January, 1997, it was observed that its presence in the mangrove area is not regular: the smallest individuals are more abundant during late summer and in autumn, and the largest ones during spring and early summer. Its diet comprises plant material and invertebrates, specially polychaets. The morphological aspects of the gonads, the monthly changes on the Condition Factor, and the monthly distribution of the individual size groups, suggest that this population spawns during the spring, out the estuarine region. The smaller individuals use the mangrove area of Guaratuba Bay to a growth phase, and the adults to make somatic reserves to the spawning period.

KEY WORDS. Diapterus rhombeus, Gerreidae, Guaratuba, Paraná, Brazil
\end{abstract}

A caratinga ou carapeba, Diapterus rhombeus (Cuvier, 1829), é um Gerreidae muito conhecido nos ambientes costeiros do sul e sudeste do Brasil. Estima-se que os adultos desovam em regiões de maior profundidade, mas que os jovens utilizam as águas rasas de praias e canais de mangue como criadouro (MENEZES \& FigueIREDO 1980). Na ictiofauna de fundo da Baía de Guaratuba, litoral do Estado do Paraná (2552'S;48³9'W), D. rhombeus está entre as espécies de presença mais comum, ocorrendo de forma abundante junto a Eucinostomus argenteus (Baird \& Girard, 1854), E. gula (Cuvier, 1830), e a representantes de Ariidae, Haemulidae, Sciaenidae, Pleuronectiformes e Tetraodontiformes. A região pode ser considerada um ambiente estuário-manguezal, em que a maior perturbação ainda se restringe às pescas artesanal e turística, ambas realizadas de maneira empírica. O porte que $D$. rhombeus atinge ali é normalmente pequeno, mas sua presença numericamente expressiva indica que ela deve desempenhar um papel relevante para a dinâmica deste sistema. Objetivando disponibilizar informações a este respeito, apresentamse a seguir dados relativos à estrutura populacional, dieta e hábitos gerais da espécie na região.

1) Contribuição número 990 do Departamento de Zoologia, Universidade Federal do Paraná. Trabalho realizado com auxilios do CNPq e Funpar.

2) Departamento de Zoologia, Universidade Federal do Paraná. Caixa Postal 19020, 81531-990 Curitiba, Paraná, Brasil.

3) Bolsista do CNPq.

4) Bolsista de Aperfeiçoamento CNPq. 


\section{MATERIAL E MÉTODOS}

Foram analisados 874 exemplares de Diapterus rhombeus, coletados mensalmente entre julho de 1993 e janeiro de 1997 (exceto maio/95), através de arrasto de fundo com portas, na área de mangue e região central da Baía de Guaratuba (Chaves \& Vendel 1997). Procedimentos biométricos, tais como mensuração de comprimento total e peso, tiveram início após conservação do material em gelo por cerca de três horas.

Uma curva peso/comprimento foi elaborada a partir dos dados provenientes de 849 indivíduos, capturados até novembro/96. Com uso do logaritmo desses valores, a regressão foi linearizada, e o coeficiente angular da reta obtida utilizado para determinação do Fator de Condição médio mensal (K), calculado individualmente segundo VAzzoler (1996): K = PT/CT ${ }^{\text {b }}$; sendo: PT o peso do exemplar, CT seu comprimento total e b o coeficiente angular da equação peso/comprimento.

Analisaram-se também o sexo e o estágio de maturação de 129 exemplares cujas gônadas puderam ser visualizadas. Teve-se a precaução de investigar os indivíduos de maior porte e em todas as épocas do ano, para evitar erros de avaliação por tratamento de exemplares jovens ou "em repouso" reprodutivo, sensu VAzZOLER (1996). Consideraram-se como "Em reprodução" os indivíduos que estavam ou "maduros", ou "em recuperação pós-desova". A primeira situação foi diagnosticada através do exame macroscópico de gônadas, conforme descrição comum a outros Perciformes (CHAves 1995). A segunda, com auxílio de preparações histológicas de ovários, de acordo com a técnica de rotina para coloração HematoxilinaEosina, e que permitiram a investigação sobre o tecido conjuntivo, seu grau de vascularização, e sobre a ocorrência nos ovários de folículos atrésicos e vazios, baseando-se em descrições já disponíveis para a família Gerreidae (CHAVEs 1989).

Para os estudos sobre dieta, estômagos de 44 indivíduos, comprimento entre 97 e $174 \mathrm{~mm}$, coletados ao longo de todo o período de trabalho, foram fixados em solução de formalina a $10 \%$. Os itens do conteúdo foram analisados em microscópios biológico e estereoscópico, e os dados obtidos tratados pelos métodos Freqüência de Ocorrência (porcentagem de estômagos contendo determinado item, em relação ao total de estômagos que continham alimento), e de Contagem de Pontos (porcentagem média de pontos ocupados por determinado item numa superfície plana quadriculada, em relação à superfície total de conteúdo). Para a interpretação comparativa sobre o grau de participação de cada item na dieta, em 37 indivíduos os resultados dos dois métodos foram conjugados no Índice de Preponderância, assim definido por NATARAJAN \& JHINGRAN (1961 apud JURAS \& YAMAGUTI 1985): IP = (FOi X Pi)/ $/ \Sigma($ FOi x POi) x 100; sendo: FOi o valor de freqüência de ocorrência e $\mathbf{P i}$ o valor da contagem de pontos para cada um dos $\mathbf{i}$ itens identificados.

A tabela I apresenta o número de exemplares computados segundo o método, a faixa de tamanho e a estação de coleta considerados, tendo-se estabelecido como de "inverno" as coletas realizadas em julho, agosto e setembro, e assim sucessivamente. 
Tabela I. Número de exemplares de $D$. rhombeus cujo conteúdo estomacal foi analisado, segundo a estação (período de referência: julho/93 a janeiro/97) e o tratamento metodológico recebido. F.O.: apenas método Freqüência de Ocorrência; I.P.: Índice de Preponderância. Acrescentam-se os comprimentos do menor e do maior exemplar trabalhados em cada situação.

\begin{tabular}{lcccc}
\hline \multicolumn{1}{c}{ Estação } & F.O. & CT min.-máx. $(\mathrm{mm})$ & I.P. & CT min.-máx. $(\mathrm{mm})$ \\
\hline Inverno & 0 & - & 10 & $99-174$ \\
Primavera & 0 & - & 18 & $97-120$ \\
Verão & 0 & - & 4 & $125-133$ \\
Outono & 7 & $102-118$ & 5 & $130-141$ \\
\hline
\end{tabular}

\section{RESULTADOS}

A abundância com que $D$. rhombeus ocorreu no manguezal durante o período de estudo não se manifestou determinada por influência sazonal. Comparados entre si os valores de captura total segundo o mês (período julho 1993 a novembro 1996), nenhuma estação se distinguiu das demais por apresentar maior ou menor quantidade de indivíduos. As capturas atingiram valores relativamente altos tanto no inverno (agosto), como na primavera (novembro) e no outono (maio); e valores relativamente baixos tanto na primavera (outubro), como no verão (março) e no outono (junho) (Fig. 1).

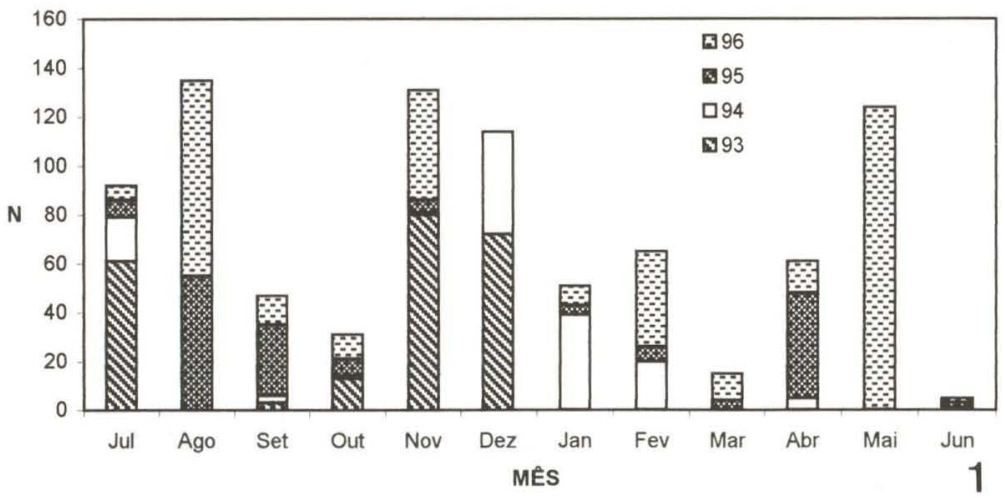

Fig. 1. Abundância mensal de Diapterus rhombeus nas amostragens realizadas na região de mangue da Baia de Guaratuba, em número de indivíduos $(\mathrm{N})$, segundo o ano. Período de referência: julho de 1993 a novembro de 1996.

O menor e o maior exemplar registrados nas coletas possuíam, respectivamente, 58 e $205 \mathrm{~mm}$. A distribuição mensal de tamanho revela que os menores indivíduos estão relativamente mais representados ao final do verão e no outono, enquanto os maiores durante a primavera e no início do verão (Fig. 2a,b).

A relação entre peso e comprimento dos indivíduos da população é visualizada na figura 3, e sua logaritimização originou a equação $\ln \mathrm{PT}=-\mathbf{1 2 , 8 7}+\mathbf{3 , 3 6}$ In CT $(r=0,98)$. O Fator de Condição, utilizando o valor 3,36 como expoente do denominador, apresentou médias mais elevadas em julho e em dezembro, e mais baixas em outubro e em junho (Fig. 4). 

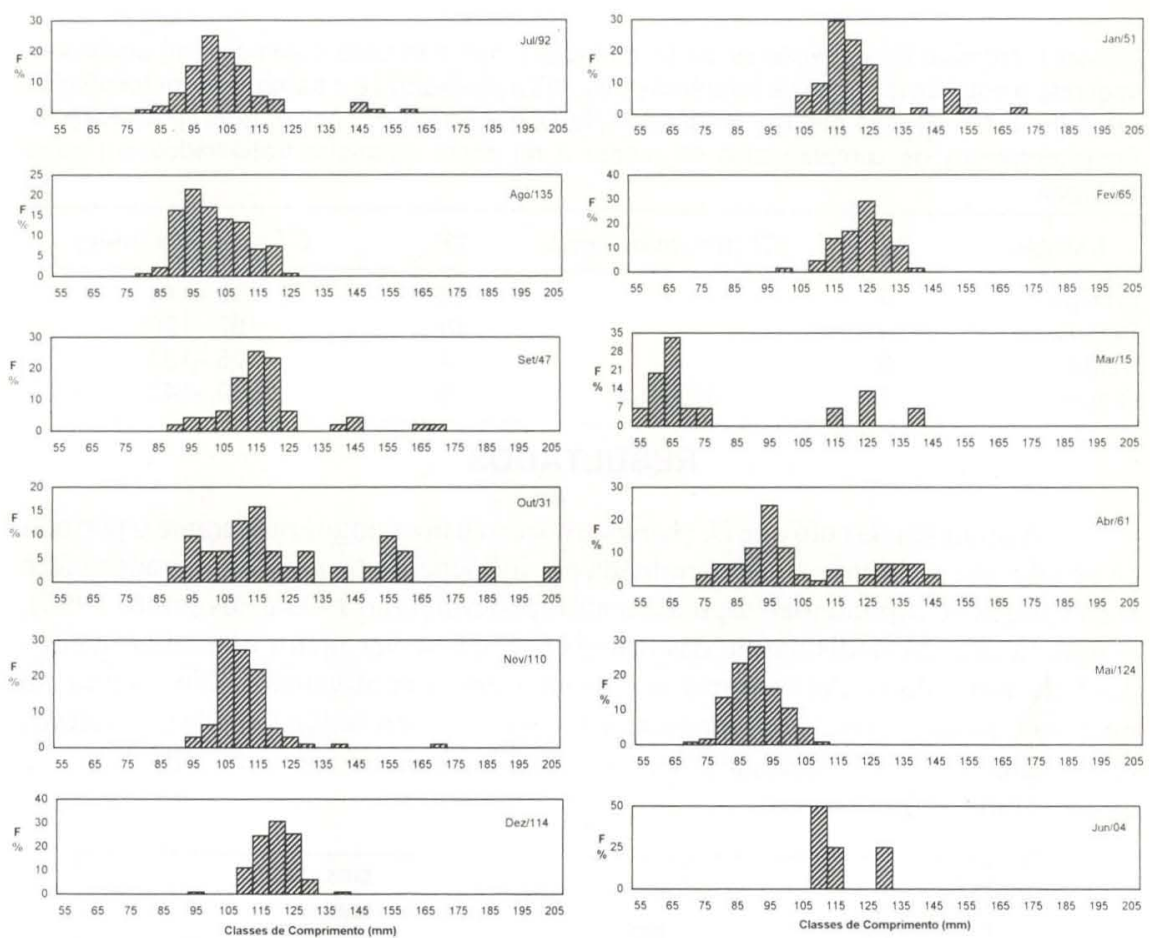

Fig. 2. Distribuição mensal da freqüência de ocorrência (\%) das classes de comprimento dos exemplares de $D$. rhombeus coletados no periodo julho/93 a novembro/96. Ao lado da indicação dos meses, registra-se o número de exemplares analisados. (A) Julho a dezembro; (B) janeiro a junho.

A atividade reprodutiva em $D$. rhombeus foi detectada apenas em dezembro e em abril (Fig. 5). No primeiro mês, gônadas maduras e ovários portando sinais histológicos de recuperação pós-desova foram registrados em quase $40 \%$ dos exemplares examinados, e no segundo em menos que $10 \%$. Folículos vazios não foram encontrados nas preparações. Constatou-se que indivíduos com tamanho inferior a $150 \mathrm{~mm}$ já podem apresentar evidências de desova anterior (Fig. 5).

Quanto à dieta, verificou-se que na Baía de Guaratuba a espécie alimenta-se de invertebrados, de vegetais, e em pequeno grau também de peixes (Tabs II, III). O item básico de sua dieta, porém, são os poliquetas, presentes em mais que $75 \%$ dos exemplares analisados e ocupando, em média, $50 \%$ do volume dos conteúdos (Tab. III). Dentre esses, foram reconhecidas a espécie Glycinde multidens Fritz Müller, 1858 (Família Goniadidae) e a família Capitellidae Grube, 1862. Crustáceos, moluscos, lofoforados e peixes também estão representados, mas partilhando com vegetais um grau de participação inferior ao de poliquetas. Nematóides ocorreram em mais que $20 \%$ dos exemplares, porém não foram considerados participantes da dieta. Cristais de areia foram registrados em 83,8\% dos conteúdos analisados (Tab. III). 

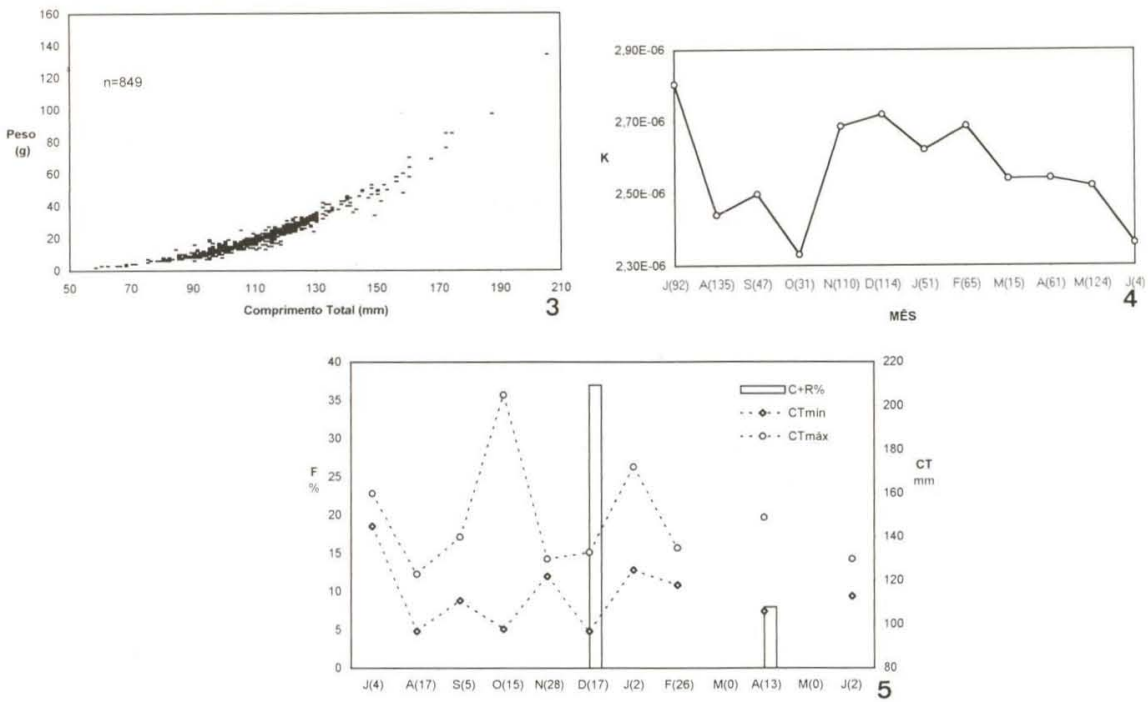

Figs 3-5. Baia de Guaratuba, período de referência: julho de 1993 a novembro de 1996. (3) Distribuição de pontos referentes à relação peso/comprimento de 849 exemplares de $D$. rhombeus; (4) variaçăo mensal média do Fator de Condição $(K)$ de $D$. rhombeus (entre parênteses, registra-se o número de indivíduos considerados em cada mês); (5) freqüência porcentual média de ocorrência $(F)$ de indivíduos considerados "em reprodução" ( $C+R \%)$, segundo o mês, em $D$. rhombeus. As curvas superior e inferior registram, respectivamente, o comprimento (CT) do maior (CTmáx) e do menor (Ctmin) exemplar considerado em cada mês. Entre parênteses, o tamanho da amostra.

Tabela II. Itens registrados no conteúdo estomacal de sete exemplares de $D$. rhombeus, cujos dados foram tratados apenas pelo método Freqüência de Ocorrência (F.O.), e seus respectivos valores.

\begin{tabular}{llll}
\hline \multicolumn{1}{c}{ Item } & F.O. & \multicolumn{1}{c}{ Item } & F.O. \\
\hline Bivalves & 42,8 & Escamas & 14,3 \\
Gamarideos & 28,6 & Poliquetas & 14,3 \\
Braquiuros & 14,3 & Detritos & 14,3 \\
Ostrácodes & 14,3 & Material não identificado & 14,3 \\
\hline
\end{tabular}

Tabela III. Itens registrados no conteúdo estomacal de 37 exemplares de $D$. rhombeus cujos dados de Freqüência de Ocorrência (F.O.) e Contagem de Pontos (P.) foram conjugados através do Índice de Preponderância (I.P.), e seus respectivos valores. "n-i": não-identificado. "P-I": pós-larvas. "Vegetais sup.": vegetais superiores.

\begin{tabular}{lrrrlrrr}
\hline \multicolumn{1}{c}{ Item } & F.O. & P. & I.P. & \multicolumn{1}{c}{ Item } & F.O. & P. & I.P. \\
\hline Poliquetas & 75,7 & 30,5 & 50,4 & Gastrópodes & 5,4 & 0,2 & $<0,1$ \\
Bivalves & 21,6 & 11,9 & 5,6 & Crustáceos n-i & 2,7 & 0,2 & $<0,1$ \\
Gamarídeos & 48,6 & 2,0 & 2,1 & Caprelídeos & 2,7 & 1,0 & $<0,1$ \\
Nematóides & 21,6 & 2,9 & 1,4 & P-I de Braquiuros & 2,7 & 0,2 & $<0,1$ \\
Copépodes & 27,0 & 1,7 & 1,0 & Vegetais sup. n-i & 37,8 & 4,1 & 3,4 \\
Phoronida & 16,2 & 2,5 & 0,9 & Carne branca n-i & 27,0 & 24,3 & 14,3 \\
Algas & 16,2 & 1,2 & 0,5 & Material não-identificado & 48,6 & 12,2 & 12,9 \\
Escamas & 10,8 & 1,2 & 0,3 & Cristais de areia & 83,8 & 3,9 & 7,1 \\
\hline
\end{tabular}




\section{DISCUSSÃO}

O padrão de abundância numérica com que $D$. rhombeus foi registrada na Baía de Guaratuba, não tendo apresentado variações de natureza sazonal, não condiz com aquele referido por M.F. Corrêa (comunicação pessoal) em relação à Baía de Paranaguá, local em que a espécie é mais comum durante o verão. Em Guaratuba observou-se, por exemplo, que considerados em conjunto os quatro invernos amostrados, a quantidade total de indivíduos capturados não foi inferior àquela registrada no conjunto dos verões ou dos outonos. É provável, no entanto, que em biomassa a influência sazonal se manifeste, consequiência da variação constatada no tamanho dos indivíduos.

A ocorrência mais expressiva de exemplares com pequeno porte no final do verão e no outono, associada às informações obtidas pela análise gonadal, indica que $D$. rhombeus desova especialmente durante a primavera. Os meses de verão possivelmente são utilizados pelos juvenis para crescimento dentro do manguezal, acompanhando a rotina de outros peixes da região (CHAVES 1994; CHAVES 1995). As condições favoráveis à alimentação na população como um todo refletem-se também sobre o Fator de Condição, que nesta época adquire valores mais altos. A elevada média registrada em julho, por sua vez, coincide com a observação pertinente a exemplares coletados naquele mês, quando apresentavam grande quantidade de gordura abdominal. Tal reserva energética provavelmente esteja relacionada à futura maturação gonadal, preparatória para a desova na primavera.

Quanto ao local em que a desova ocorre, não há evidências que apontem para o interior da Baía. As observações aqui realizadas indicam que os exemplares de menor porte residem no manguezal durante uma etapa de seu crescimento, enquanto os de maior porte armazenam, também neste ambiente, reservas somáticas para o período reprodutivo. A desova, em si, possivelmente ocorra no ambiente oceânico, em águas profundas, conforme citado por MENEZES \& FIGUEIREDo (1980). A Baía de Guaratuba representaria para a espécie um local de residência temporária, conclusão a que também chegaram TEIXEIRA \& FALCÃo (1992) com respeito à população de $D$. rhombeus de um ambiente lagunar do nordeste brasileiro.

Os dados obtidos com relação à dieta da espécie na região condizem com os referidos por MENEZES \& FigUEIREDO (1980), de que D. rhombeus alimenta-se de algas e pequenos invertebrados. A participação dominante de poliquetas possivelmente está associada à elevada freqüência de ocorrência de sedimento no conteúdo estomacal, fato que mostra a existência de uma influência direta do leito da região estuarina sobre os hábitos desta espécie. Crustáceos decápodes, que observações de CHAVES \& VENDEL (1996) revelaram ser tão comuns na dieta de um outro teleósteo do manguezal - Genidens genidens (Valenciennes, 1839), têm em D. rhombeus presença muito discreta. Quanto ao item peixes, sua participação em volume é certamente superior àquela quantificada por suas escamas, pois parte da "carne branca não-identificada" deve referir-se a tal item. Todavia, a julgar pela freqüência inferior a $11 \%$ com que são registrados no conteúdo, os peixes efetivamente contribuem para a dieta de $D$. rhombeus em grau muito inferior ao dos invertebrados e vegetais. 
AGRADECIMENTOS. À Profa Rosana Moreira da Rocha e aos biólogos Ronny Scheffler de Moura e Janete Dubiaski Silva, Universidade Federal do Paraná, pelas orientações prestadas no reconhecimento de itens do conteúdo estomacal. Ainda, a dois Revisores anônimos, pela análise do texto e contribuição à versão final.

\section{REFERÊNCIAS BIBLIOGRÁFICAS}

Chaves, P.T.C. 1989. Desenvolvimento dos ovócitos em Harengula clupeola, Urophycis brasiliensis, Eucinostomus argenteus, Isopisthus parvipinnis e Menticirrhus americanus (Teleostei). Bolm Inst. oceanogr., São Paulo, 37 (2): 81-93.

1994. A incubação de ovos e larvas em Genidens genidens (Valenciennes) (Siluriformes, Ariidae) da Baía de Guaratuba, Paraná, Brasil. Revta bras. Zool. 11 (4): $641-648$.

1995. Atividade reprodutiva de Bairdiella ronchus (Cuvier) (Pisces, Sciaenidae) na Baía de Guaratuba, Paraná, Brasil. Revta bras. Zool. 12 (4): 759-766.

Chaves, P.T.C. \& A.L. Vendel. 1996. Aspectos da alimentação de Genidens genidens (Valenciennes) (Siluriformes, Ariidae) na Baía de Guaratuba, Paraná. Revta bras. Zool. 13 (3): 669-675.

1997. Indicadores reprodutivos das espécies de Citharichthys Bleeker (Teleostei, Pleuronectiformes) na Baía de Guaratuba, Paraná, Brasil. Revta bras. Zool. 13 (3): 669-675.

JURAS, A.A. \& N. YAMAGUTI. 1985. Food and feeding habits os king weakfish, Macrodon ancylodon (Bloch \& Schneider, 1801) caught in the Southern Coast of Brazil (Lat. $29^{\circ} \mathrm{S}$ to $32^{\circ} \mathrm{S}$ ). Bolm Inst. oceanogr., São Paulo, 33 (2): 149-157. Menezes, N.A. \& J.L. Figueiredo. 1980. Manual de Peixes Marinhos do Sudeste do Brasil. IV. Teleostei (3). São Paulo, Museu de Zoologia, Universidade de São Paulo, 96p.

TEIXEIRA, R.L. \& G.A.F. FALCÃo. 1992. Composição da fauna nectônica do Complexo Lagunar Mundaú/Manguaba, Maceió - AL. Atlântica, Rio Grande, 14: 43-58.

Vazzoler, A.E.A. DE M. 1996. Biologia da Reprodução de Peixes Teleósteos: Teoria e Prática. Maringá, EDUEM/SBI, 169p.

Recebido em 20.I.1997; aceito em 13.IV.1998. 